\title{
Comparison of lentiviruses pseudotyped with S proteins from coronaviruses and cell tropisms of porcine coronaviruses
}

\author{
Jingjing Wang ${ }^{1,2}$, Feng Deng ${ }^{1,2}$, Gang Ye ${ }^{1,2}$, Wanyu Dong ${ }^{1,2}$, Anjun Zheng ${ }^{1,2}$, Qigai He ${ }^{1,2,3}$, \\ Guiqing Peng ${ }^{1,2,3 \bowtie}$
}

1. State Key Laboratory of Agricultural Microbiology, Huazhong Agricultural University, Wuhan 430070, China

2. College of Veterinary Medicine, Huazhong Agricultural University, Wuhan 430070, China

3. The Cooperative Innovation Center for Sustainable Pig Production, Huazhong Agricultural University, Wuhan 430070, China

The surface glycoproteins of coronaviruses play an important role in receptor binding and cell entry. Different coronaviruses interact with their specific receptors to enter host cells. Lentiviruses pseudotyped with their spike proteins (S) were compared to analyze the entry efficiency of various coronaviruses. Our results indicated that $\mathrm{S}$ proteins from different coronaviruses displayed varied abilities to mediate pseudotyped virus infection. Furthermore, the cell tropisms of porcine epidemic diarrhea virus (PEDV) and transmissible gastroenteritis virus (TGEV) have been characterized by live and pseudotyped viruses. Both live and pseudoviruses could infected VeroCCL-81 (monkey kidney), Huh-7 (human liver), and PK-15 (pig kidney) cells efficiently. CCL94 (cat kidney) cells could be infected efficiently by TGEV but not PEDV. Overall, our study provides new insights into the mechanisms of viral entry and forms a basis for antiviral drug screening.

\section{KEYWORDS Coronavirus; spike proteins; receptor binding; cell entry; pseudotyped virus}

\section{INTRODUCTION}

Coronaviruses (CoVs) are important infectious pathogens that are associated closely with respiratory and enteric diseases in humans and animals (Perlman and Netland, 2009; Belouzard et al., 2012; Li, 2013). CoVs have a single-strand positive sense RNA genome and consist of four groups: Alphacoronavirus, Betacoronavirus, Gammacoronavirus, and Deltacoronavirus (Table 1).

Viral entry into cells is highly dependent on the interaction between viral particles and the host cells. CoVs use a variety of cellular receptors and co-receptors, including proteins and sugars, to facilitate their entry into cells. Infection begins with an interaction between the virus and its specific receptors $(\mathrm{Li}, 2015)$. Severe acute Received: 26 November 2015, Accepted: 28 January 2016, Published online: 19 February 2016 $\triangle$ Correspondence: Phone: +86-18071438015, Email: penggq@mail.hzau.edu.cn ORCID: 0000-0001-5419-2499 respiratory syndrome coronavirus (SARS-CoV) of group beta uses a type I integral membrane protein, angiotensin-converting enzyme 2 (ACE2), as a receptor ( $\mathrm{Li}$ et al., 2005). There is no structural homology between SARS-CoV and the Human coronavirus NL63 (HCoVNL63) receptor-binding domain (RBD), but they recognize the same receptor, ACE2 (Wu et al., 2011). Although the RBD crystal structures of transmissible gastroenteritis virus (TGEV) and HCoV-NL63 are similar, they use different receptors (Reguera et al., 2012). Aminopeptidase N (APN), also known as CD13, is a type II transmembrane protein (Tusell et al., 2007). TGEV and porcine epidemic diarrhea virus (PEDV) infect cells through interaction with porcine (p) APN (Li et al., 2007; Nam and Lee, 2010). Human (h) APN is a receptor for HCoV-229E (Belouzard et al., 2012). In the same beta group, the receptors for mouse hepatitis virus (MHV) and bovine coronavirus (BCoV) are carcinoembryonic antigen-related cell adhesion molecule 1 (CEACAM1) and a sugar, respectively, despite their 
Table 1. Coronavirus genera, species, and receptors.

\begin{tabular}{lll}
\hline Genus & Species & Receptor, co-receptor \\
\hline Alphacoronavirus & HCoV-229E & hAPN \\
& HCoV-NL63 & ACE2 \\
& TGEV & pAPN, Neu5Ac, Neu5Gc \\
Betacoronavirus & PEDV & pAPN, Neu5Ac \\
& SARS-CoV & ACE2 \\
& MHV & CEACAM1 \\
& BCoV & Neu5, 9Ac2 \\
Gammacoronavirus & HCoV-OC43 & Neu5, 9Ac2 \\
Deltacoronavirus & IBV & Neu5Ac \\
& Bulbul coronavirus HKU11 & Unknown \\
\hline
\end{tabular}

high sequence homology (Peng et al., 2011; Peng et al., 2012). CEACAM1, the first identified coronavirus receptor (Dveksler et al., 1991), is a type I transmembrane multifunctional protein of the immunoglobulin superfamily (de Haan et al., 2005).

Cell entry and interspecies transmission of CoVs are mediated by the spike protein (S). CoV S is a class I fusion protein (Bosch et al., 2003). In CoVs, $\mathrm{S}$ is a significant surface protein and plays an important role in mediating infection of virions (Schwegmann-Wessels et al., 2009). S is also responsible for receptor binding and the fusion of viral and cellular membranes. All CoV S share the same functional component in two domains: an $\mathrm{N}$ terminal domain called $\mathrm{S} 1$ that is responsible for receptor binding, and a C-terminal S2 domain that is responsible for fusion. S1 contains two subdomains, an N-terminal domain and a C-terminal domain (Figure 1A). Both function as RBDs and bind a variety of proteins and sugars (Belouzard et al., 2012).

Because some CoVs have strong pathogenicity, the lentiviral pseudotype system is a reliable tool to study the proteins of highly pathogenic viruses under conventional biosafety conditions. HIV-Luc is an HIV-1 based lentiviral vector bearing the luciferase reporter gene and has been used in the production of pseudotyped viruses (Kang et al., 2012; Lu et al., 2012; Tang et al., 2012). Pseudovirus entry efficiency is characterized based on the luciferase activity. Viral particles pseudotyped by various $\mathrm{S}$ proteins have been described for several viruses. In our study, we compared the efficiency of pseudotyped viruses with $\mathrm{S}$ proteins from different groups of CoVs. Furthermore, the cell tropisms of TGEV and PEDV were characterized by live and pseudotyped viruses.

\section{MATERIALS AND METHODS}

\section{Cell lines, virus strains and antibodies}

293 T cells were cultured and maintained in RPMI 1640 medium (Invitrogen, Carlsbad, CA, USA) supplemented with $10 \%$ fetal bovine serum (FBS) at $37{ }^{\circ} \mathrm{C}$ in an atmosphere of $5 \% \mathrm{CO}_{2}$. PK-15, Huh-7, Vero-CCL-81, MDBK, CCL94, BSR, and MDCK cells were cultured and maintained in Dulbecco's Modified Eagle's modium (DMEM) (Invitrogen) supplemented with 10\% fetal bovine serum (FBS) at $37{ }^{\circ} \mathrm{C}$ in an atmosphere of $5 \%$ $\mathrm{CO}_{2}$. The PEDV and TGEV strains (GenBank accession numbers: KT021232.1 for PEDV, HQ462571.1 for TGEV) were isolated from a suckling piglet. Rabbit antiPEDV N protein and rabbit anti-TGEV N protein polyclonal antibodies are stored by the lab.

\section{Plasmids}

The $\mathrm{S}$ protein sequences of different groups of CoVs (GenBank accession numbers: ABD72982.1 for SARSCoV-S, AAR92025.1 for MHV-S, NP 073551.1 for HCoV229E-S, ABG89335.1 for TGEV-S, NP 598310.1 for PEDV-S, AAT84362.1 for HCoV-OC43-S, ABM66810.1 for BCoV-S, and NP 040831.1 for avian infectious bronchitis virus (IBV) $\overline{\mathrm{S}}$ ) were codon-optimized and synthesized (GenScript, Nanjing, China). The S fragments were cloned into the pcDNA3.1 $(+)$ vector with a C-terminal His-tag.

\section{Production of lentiviruses pseudotyped with S proteins from CoVs of different groups}

On the day before transfection, 293T cells were seeded in six-well plates. The next day, the cells were transfected with S plasmids plus the lentiviral vector HIV-Luc 
A

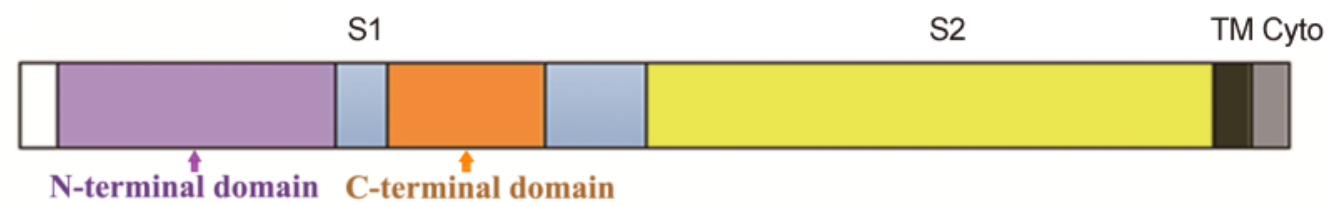

B Amino acid sequence identities of $\mathrm{S}$ proteins from coronaviruses of different groups

\begin{tabular}{|c|c|c|c|c|c|c|c|c|}
\hline & 229E-S(\%) & TGEV-S(\%) & PEDV-S(\%) & SARS-CoV-S(\%) & MHV-S(\%) & OC43-S(\%) & $\mathrm{BCoV}-\mathrm{S}(\%)$ & IBV-S(\%) \\
\hline IBV-S & 26.2 & 25.1 & 25.4 & 22.3 & 22.5 & 23.5 & 23.5 & - \\
\hline BCoV-S & 24.9 & 22.1 & 20.8 & 28.1 & 64.5 & 99.8 & - & \\
\hline OC43-S & 24.9 & 22.1 & 20.8 & 28.1 & 64.5 & - & & \\
\hline MHV-S & 22.0 & 21.1 & 19.7 & 26.6 & - & & & \\
\hline SARS-CoV-S & 24.0 & 21.6 & 21.5 & - & & & & \\
\hline PEDV-S & 49.2 & 45.1 & - & & & & & \\
\hline TGEV-S & 49.4 & - & & & & & & \\
\hline $229 \mathrm{E}-\mathrm{S}$ & - & & & & & & & \\
\hline
\end{tabular}

C Amino acid sequence identities of S1 proteins from coronaviruses of different groups

\begin{tabular}{|c|c|c|c|c|c|c|c|c|}
\hline & 229E-S1(\%) & TGEV-S1(\%) & PEDV-S1(\%) & SARS-CoV-S1(\%) & MHV-S1(\%) & OC43-S1(\%) & BCoV-S1(\%) & IBV-S1(\%) \\
\hline IBV-SI & 11.3 & 9.3 & 10.6 & 9.8 & 6.9 & 8.8 & 8.5 & - \\
\hline BCoV-S1 & 9.8 & 11.5 & 10.1 & 16.0 & 59.4 & 98.3 & - & \\
\hline OC43-S1 & 9.6 & 11.5 & 10.1 & 15.8 & 59.7 & - & & \\
\hline MHV-S1 & 7.8 & 10.3 & 10.5 & 17.3 & - & & & \\
\hline SARS-CoV-S1 & 10.5 & 9.7 & 10.0 & - & & & & \\
\hline PEDV-S1 & 35.9 & 30.3 & - & & & & & \\
\hline TGEV-S1 & 35.6 & - & & & & & & \\
\hline 229E-S1 & - & & & & & & & \\
\hline
\end{tabular}

Figure 1. S proteins from coronaviruses of different groups. (A) Domain structure of the $\mathrm{S}$ protein, including the $\mathrm{S} 1$ and $\mathrm{S} 2$ regions, transmembrane domain (TM), and cytoplasmic tail (Cyto). (B) Amino acid sequence identities of $S$ proteins from coronaviruses of different groups. The GenBank accession numbers are as follows: ABD72982.1 for SARS-CoV-S, AAR92025.1 for MHV-S, NP_073551.1 for HCoV-229E-S, ABG89335.1 for TGEV-S, NP_598310.1 for PEDV-S, AAT84362.1 for HCoV-OC43-S, ABM66810.1 for BCoV-S, and NP_040831.1 for IBV-S. (C) Amino acid sequence identities of $\mathrm{S} 1$ proteins from coronaviruses of different groups.

using the Lipofectamine 2000 (Invitrogen) transfection reagent. After incubation for $5-6 \mathrm{~h}$ at $37^{\circ} \mathrm{C}$, the supernatant of cultured cells was replaced by DMEM containing $2 \%$ FBS. After $48 \mathrm{~h}$, the supernatants of transfected cells containing pseudotyped viruses were harvested, and the cell debris was removed by low-speed centrifugation. The pseudoviruses were quantified with an HIV-1 p24 EIA kit (Beckman-Coulter, Brea, CA, USA).

\section{Western blot analysis of the pseudovirus} construction

Lentiviruses pseudotyped with S proteins were solubilized by boiling in sodium dodecyl sulfate sample buffer. The $\mathrm{S}$ proteins were fractionated by sodium dodecyl sulfate- $10 \%$ polyacrylamide gel electrophoresis and transferred to a nitrocellulose membrane. The nitrocellulose membrane was incubated with a 1:4000 dilution of a 
mouse anti-His antibody (ProteinTech Group, Chicago, IL, USA). Goat anti-mouse IgG (Boster, Wuhan, China) was used as the secondary antibody.

\section{Expression of receptors}

A plasmid (Vector pcDNA 3.1(+)) with each viral receptor gene (ACE2, CEACAM1, hAPN, and pAPN) was transfected into 293T cells with Lipofectamine 2000. After incubation for $5-6 \mathrm{~h}$ at $37^{\circ} \mathrm{C}$, the supernatant was replaced by DMEM containing 2\% FBS. After $48 \mathrm{~h}$, the cells were infected by lentiviruses pseudotyped with the $\mathrm{S}$ proteins from $\mathrm{CoVs}$ of different groups.

\section{Infection of lentiviruses pseudotyped with S proteins}

For analysis of the lentiviruses pseudotyped with the $\mathrm{S}$ proteins from CoVs of different groups, different cell lines were seeded in 96-well plates. 293T cells expressing receptors were infected by the same lentiviruses and incubated at $37^{\circ} \mathrm{C}$ for $2 \mathrm{~h}$. DMEM $(150 \mu \mathrm{L})$ containing $2 \%$ FBS was added subsequently to all cells and incub- ated for an additional $48-72 \mathrm{~h}$. The pseudovirus entry efficiency was characterized based on luciferase activity.

\section{Cell lines were infected by PEDV and TGEV}

PEDV and TGEV were used to infect various cell lines from different species, including PK-15 (pig kidney), Huh-7 (human liver), Vero-CCL-81 (monkey kidney), MDBK (bovine kidney), CCL94 (cat kidney), BSR (hamster kidney), and MDCK (canine kidney) cells at a multiplicity of infection of 0.01 . Trypsin $(10 \mu \mathrm{g} / \mathrm{mL})$ was included in the cell culture medium to facilitate live virus infections. Cells infected with viruses were fixed with $4.0 \%(\mathrm{v} / \mathrm{v})$ paraformaldehyde at 24 or $48 \mathrm{~h}$ post-inoculation. PEDV or TGEV was detected with fluorescein isothiocyanate-labeled rabbit anti-PEDV or anti-TGEV N protein antibodies, respectively, and observed under a fluorescence microscope.

\section{Statistical analyses}

All experiments were done at least twice (in most cases three or more times). Data were analyzed statistically using two-tailed student's $t$-tests for comparison of pcDNA3.1 and CoV S pseudovirus.

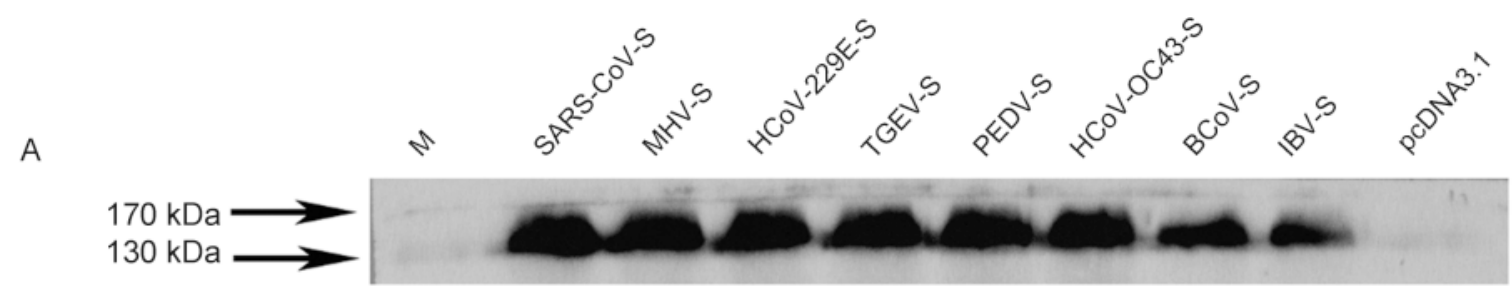

B

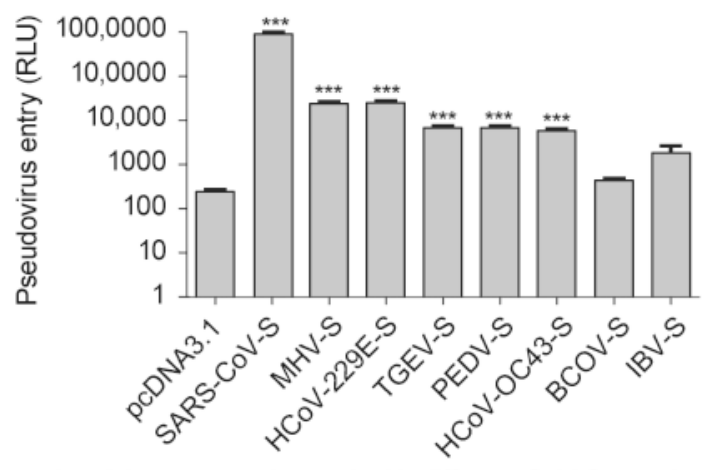

C

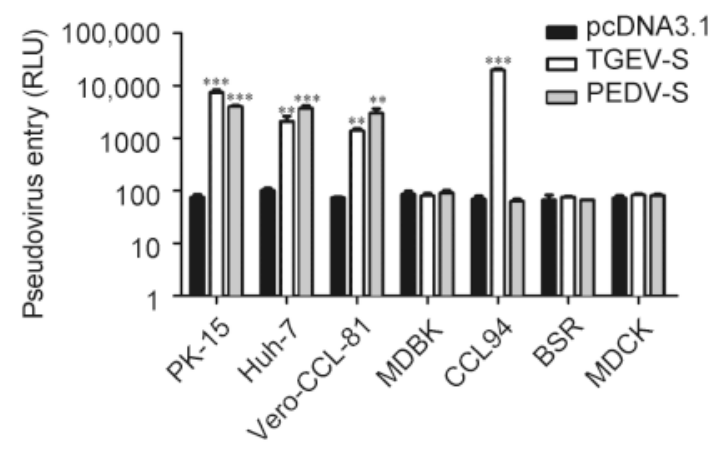

Cell lines

Figure 2. Coronavirus-spike-mediated entry of pseudoviruses into host cells. (A) Western blotting of the $S$ protein of pseudoviruses. (B) Lentiviruses pseudotyped with different coronavirus $S$ proteins were generated and used to infect different cells. SARS-CoV-S pseudoviruses infected 293T cells expressing endogenous ACE2. TGEV-S and PEDV-S pseudoviruses infected 293T cells expressing endogenous PAPN. MHV-S and HCoV-229E-S pseudoviruses entered cells expressing CEACAM1 and hAPN, respectively. BCoV-S, HCoV-OC43-S, and IBV-S infected 293T cells directly. (C) TGEV-S and PEDV-S pseudoviruses infected PK-15, Huh-7, Vero-CCL-81, MDBK, CCL94, BSR, and MDCK cells. The pseudovirus entry efficiency is reported as the luciferase activity. Statistical analyses were performed to compare the treatment groups: ${ }^{* \star *} P<0.001 ;{ }^{* *} 0.001<P<0.01$. 


\section{RESULTS}

\section{Sequence alignment}

$\mathrm{CoV}$ diversity is reflected in the variable S proteins (HealdSargent and Gallagher, 2012). Therefore, we compared the $\mathrm{S}$ protein amino acid sequences of CoVs from different groups, including SARS-CoV, MHV, HCoV-229E, TGEV, PEDV, HCoV-OC43, BCoV, and IBV (GenBank accession numbers ABD72982.1, AAR92025.1, NP 073551.1, ABG89335.1, NP 598310.1, AAT84362.1, ABM66810.1, and NP_040831.1, respectively) using ClustalW (Figure 1B). Because $\mathrm{S} 1$ is responsible for receptor binding, the amino acid sequences of the S1 domain were aligned (Figure $1 \mathrm{C}$ ). The $\mathrm{S}$ proteins from $\mathrm{CoVs}$ of the same group shared sequence similarities of greater than $20 \%$, with some similarities of up to $40 \%$. The S1 sequence similarities of CoVs from different groups were less than $12 \%$.

\section{Comparison of lentiviruses pseudotyped with S proteins of CoVs}

To determine the success of pseudovirus construction, the $\mathrm{S}$ proteins of pseudoviruses were confirmed by western blotting (Figure 2A). The pseudoviruses were quantified with an HIV-1 p24 EIA kit and used to infect cells expressing the corresponding receptor. Lentiviruses pseudotyped with SARS-CoV S protein could efficiently infect $293 \mathrm{~T}$ cells expressing ACE2, and the pseudovirus level after entry reached $10^{6}$ relative light units (RLU) (Figure 2B). The S proteins of HCoV-229E, TGEV, and PEDV exhibit high homology. However, their pseudovirus infection efficiency varies, and they are approximately 50-fold less efficient than the SARSCoV-S pseudovirus. The TGEV-S and PEDV-S pseudoviruses showed similar infection efficiencies. Although $\mathrm{HCoV}-\mathrm{OC} 43$, BCoV, and IBV utilize a sugar as their receptors, their pseudovirus infection efficiency was not clear.

TGEV-S and PEDV-S pseudoviruses were used to infect PK-15, Huh-7, Vero-CCL-81, MDBK, CCL94, BSR, and MDCK cells (Figure 2C). The TGEV-S and PEDV-S pseudoviruses could enter PK-15, Huh-7, and Vero-CCL-81 cells with a similar efficiency. However, there was an intriguing finding that CCL94 cells could be infected efficiently by TGEV-S but not PEDV-S pseudoviruses.

\section{Live PEDV and TGEV have different cell tropisms}

To further study the cellular entry of CoVs, we used live PEDV and TGEV to infect different cell lines. PEDV efficiently infected Vero-CCL-81 (monkey kidney), Huh-7 (human liver), and PK-15 (pig kidney) cells (Figure 3). Infection was not evident in MDBK (bovine kidney), CCL94 (cat kidney), BSR (hamster kidney), or MDCK (canine kidney) cells. This is consistent with the data in
Figure 2C. TGEV efficiently infected cells from pigs (PK-15), humans (Huh-7), monkeys (Vero-CCL-81), and felines (CCL94) (Figure 4). Overall, our study demonstrates that PEDV and TGEV can infect cells from different species.

\section{DISCUSSION}

CoVs recognize a variety of cell-surface molecules as their host receptors, including proteins, sugars, and heparan sulfate $(\mathrm{Li}, 2013)$. The cell entry mechanisms of these viruses are mediated by the viral $\mathrm{S}$ proteins that bind cellular receptors and mediate the fusion of viral and cellular membranes (Heald-Sargent and Gallagher, 2012). We demonstrated that $\mathrm{S}$ proteins from CoVs of different groups played an important role in mediating viral infection at different efficiencies. SARS-CoV-S pseudoviruses efficiently entered cells expressing ACE2. This result is consistent with previous reports demonstrating that ACE2 is a receptor for SARS-CoV (SchwegmannWessels et al., 2009; Wu et al., 2011). HCoV-229E-S and MHV-S pseudoviruses showed strong infectivity, in part because they use proteins as a receptor (Li et al., 2005; Belouzard et al., 2012). Moreover, PEDV-S and TGEV$\mathrm{S}$ pseudoviruses had similar infection efficiencies in PK15, Huh-7, or Vero-CCL-81 cells, and TGEV-S pseudovirus-infected CCL94 cells.

The sugar moiety 5-N-acetyl-9-O-acetylneuraminic acid (Neu5, 9Ac2), found on cell-surface glycoproteins or glycolipids, is recognized by $\mathrm{BCoV}$ and $\mathrm{HCoV}-\mathrm{OC} 43$. In addition, two other types of sugars, 5-N-glycolylneuraminic acid (Neu5Gc) and 5-N-acetylneuraminic acid (Neu5Ac), can serve as receptors or co-receptors for some alpha- and gamma-CoVs (Cavanagh and Davis, 1986; Krempl et al., 1997; Peng et al., 2012; Shahwan et al., 2013). The S-to-sugar binding affinity is lower than the S-protein interaction, which partially explains why the BCoV and IBV pseudoviruses have low entry efficiency.

Although PEDV and TGEV use the same receptor (pAPN) and the S proteins present high homology, live PEDV and TGEV prefer different cell lines; PEDV prefers Vero-CCL-81, Huh-7, and PK-15 cells. This is consistent with a previous report (Liu et al., 2015). However, TGEV prefers Vero-CCL-81, Huh-7, PK-15, and CCL94 cells. This indicates that PEDV and TGEV may have different species preferences. These results are consistent with the observation that their receptor-binding domains are located in non-homologous regions (Belouzard et al., 2012). However, the mechanism underlying different cell tropisms requires further investigation.

The $\mathrm{S}$ protein plays a crucial role in entry into host cells by mediating receptor binding and membrane fusion. CoVs use a variety of receptors and triggers to ac- 

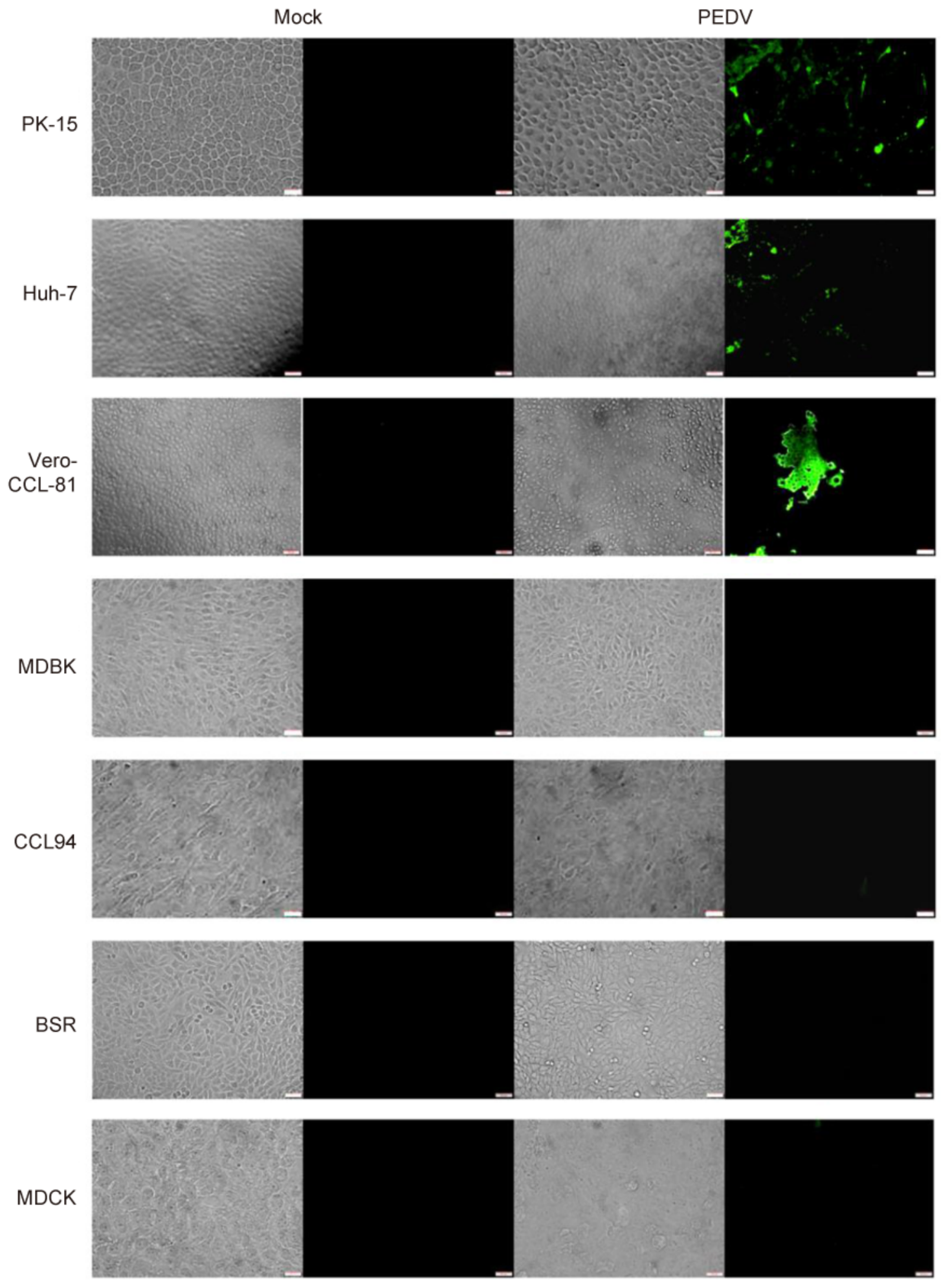

Figure 3. PEDV infections in cultured cells. The PEDV strain, YN144, was used to infect different cell lines at a multiplicity of infection of 0.01 . Trypsin $(10 \mu \mathrm{g} / \mathrm{mL})$ was included in the medium to facilitate live PEDV infection. Cells infected with PEDV were fixed with $4.0 \%(\mathrm{v} / \mathrm{v})$ paraformaldehyde at $24 \mathrm{~h}$ post-inoculation. PEDV was detected with a fluorescein isothiocyanate-labeled rabbit anti-PEDV N protein antibody and observed under a fluorescence microscope. Scale bars are $50 \mu \mathrm{m}$. 

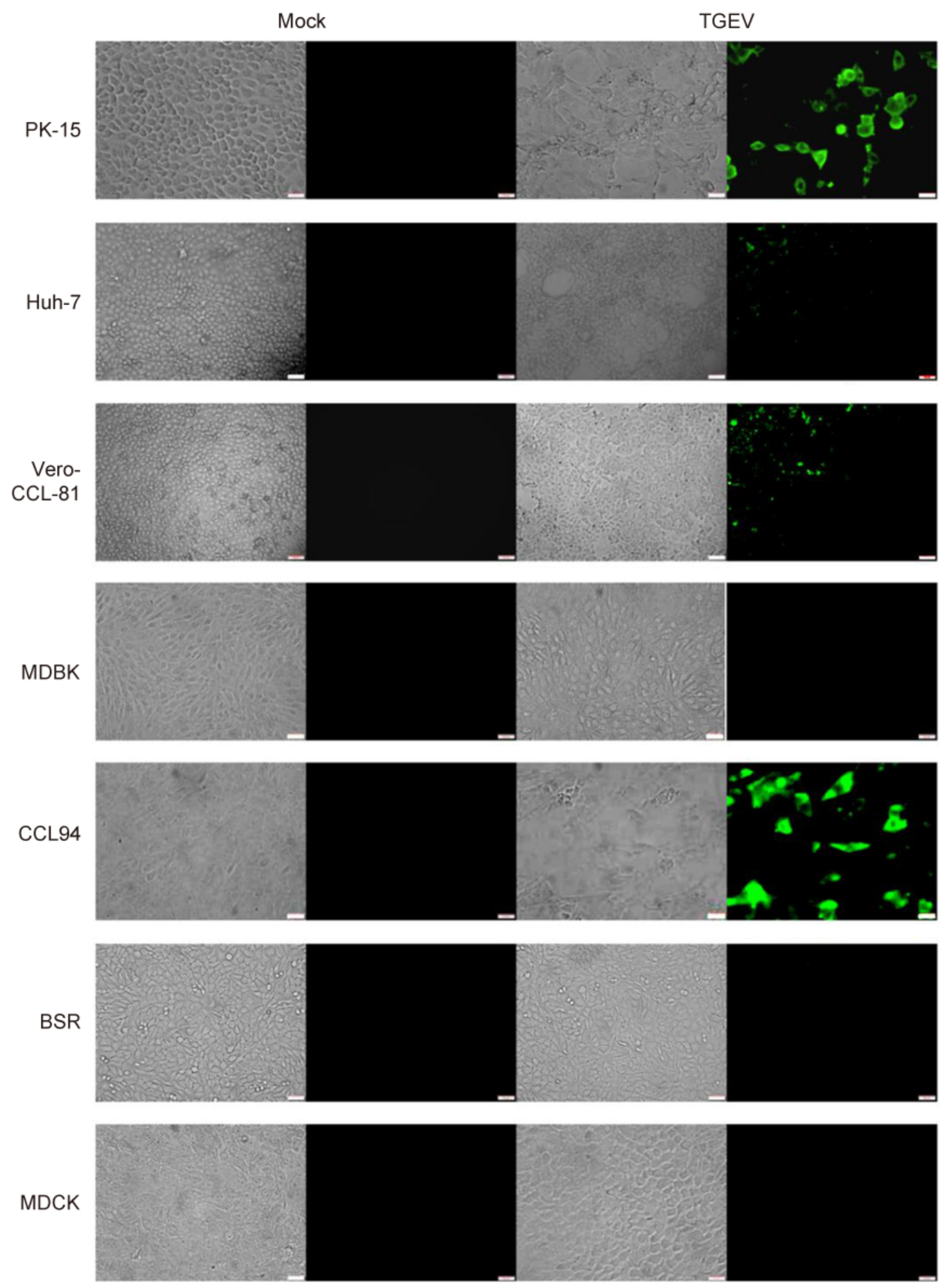

Figure 4. TGEV infections in cultured cells. The TGEV-WH-1 strain was used to infect different cell lines at a multiplicity of infection of 0.01 . Trypsin $(10 \mu \mathrm{g} / \mathrm{mL})$ was included in the medium to facilitate live TGEV infections. Cells infected with TGEV were fixed with $4.0 \%(\mathrm{v} / \mathrm{v})$ paraformaldehyde at $48 \mathrm{~h}$ post-inoculation. TGEV was detected with a fluorescein isothiocyanate-labeled rabbit anti-TGEV N protein antibody and observed under a fluorescence microscope. Scale bars are $50 \mu \mathrm{m}$. 
tivate fusion (Belouzard et al., 2012). The first and most important step of virus infection is the interaction between the virus and its cellular receptor. Infection of lentiviruses pseudotyped shows that $\mathrm{S}$ proteins from different CoVs have varied abilities to mediate pseudotyped virus infection in different cell types from different tissues. These findings further our understanding of the mechanisms underlying viral invasion and contribute to the development of drugs against CoVs.

\section{ACKNOWLEDGMENTS}

This work was supported by the National Natural Science Foundation of China (Grant No. 31372440).

\section{COMPLIANCE WITH ETHICS GUIDELINES}

This article does not contain any studies with human or animal subjects performed by any of the authors. There is no competing interest in this research.

\section{AUTHOR CONTRIBUTIONS}

G.P. and J.W. designed the experiments and wrote the paper. J.W. performed the experiments. F.D. and Q.H. contributed to critical discussions of the data. All authors approved the final manuscript.

\section{REFERENCES}

Belouzard S, Millet JK, Licitra BN, Whittaker GR. 2012. Mechanisms of coronavirus cell entry mediated by the viral spike protein. Viruses, 4: 1011-1033.

Bosch BJ, van der Zee R, de Haan CAM, Rottier PJM. 2003. The coronavirus spike protein is a class I virus fusion protein: Structural and functional characterization of the fusion core complex. J Virol, 77: 8801-8811.

Cavanagh D, Davis PJ. 1986. Coronavirus IBV: removal of spike glycopolypeptide $\mathrm{S} 1$ by urea abolishes infectivity and haemagglutination but not attachment to cells. J Gen Virol, 67: 1443-1448.

de Haan CAM, Li Z, Lintelo ET, Bosch BJ, Haijema BJ, Rottier PJM. 2005. Murine coronavirus with an extended host range uses heparan sulfate as an entry receptor. J Virol, 79: 1445114456.

Dveksler GS, Pensiero MN, Cardellichio CB, Williams RK, Jiang GS, Holmes KV, Dieffenbach CW. 1991. Cloning of the mouse hepatitis virus (MHV) receptor: expression in human and hamster cell lines confers susceptibility to MHV. J Virol, 65: 6881-6891.

Heald-Sargent T, Gallagher T. 2012. Ready, set, fuse! The coronavirus spike protein and acquisition of fusion competence. Viruses, 4: 557-580.

Kang YX, Wu ZW, Lau TCK, Lu XF, Liu L, Cheung AKL, Tan ZW, Ng J, Liang JG, Wang HB, Li SK, Zheng BJ, Li B, Chen L, Chen ZW. 2012. CCR5 antagonist TD-0680 uses a novel mechanism for enhanced potency against HIV-1 entry, cell-mediated infection, and a resistant variant. J Biol Chem, 287: 16499-16509.

Krempl C, Schultze B, Laude H, Herrler G. 1997. Point mutations in the $\mathrm{S}$ protein connect the sialic acid binding activity with the enteropathogenicity of transmissible gastroenteritis coronavirus. J Virol, 71: 3285-3287.

Li BX, Ge JW, Li YJ. 2007. Porcine aminopeptidase N is a functional receptor for the PEDV coronavirus. Virology, 365: 166172.

Li F. 2013. Receptor recognition and cross-species infections of SARS coronavirus. Antiviral Res, 100: 246-254.

Li F. 2015. Receptor recognition mechanisms of coronaviruses: a decade of structural studies. J Virol, 89: 1954-1964.

Li F, Li WH, Farzan M, Harrison SC. 2005. Structure of SARS coronavirus spike receptor-binding domain complexed with receptor. Science, 309: 1864-1868.

Liu C, Tang J, Ma Y, Liang X, Yang Y, Peng G, Qi Q, Jiang S, Li J, Du L, Li F. 2015. Receptor usage and cell entry of porcine epidemic diarrhea coronavirus. J Virol, 89: 6121-6125.

Lu X, Liu L, Zhang X, Lau TC, Tsui SK, Kang Y, Zheng P, Zheng B, Liu G, Chen Z. 2012. F18, a novel small-molecule nonnucleoside reverse transcriptase inhibitor, inhibits HIV-1 replication using distinct binding motifs as demonstrated by resistance selection and docking analysis. Antimicrob Agents Chemother, 56: 341-351.

Nam E, Lee C. 2010. Contribution of the porcine aminopeptidase $\mathrm{N}$ (CD13) receptor density to porcine epidemic diarrhea virus infection. Vet Microbiol, 144: 41-50.

Peng GQ, Sun DW, Rajashankar KR, Qian ZH, Holmes KV, Li F. 2011. Crystal structure of mouse coronavirus receptor-binding domain complexed with its murine receptor. Proceed Nat Acad Sci USA, 108: 10696-10701.

Peng GQ, Xu LQ, Lin YL, Chen L, Pasquarella JR, Holmes KV, Li F. 2012. Crystal structure of bovine coronavirus spike protein lectin domain. J Biol Chem, 287: 41931-41938.

Perlman S, Netland J. 2009. Coronaviruses post-SARS: update on replication and pathogenesis. Nat Rev Microbiol, 7: 439-450.

Reguera J, Santiago C, Mudgal G, Ordono D, Enjuanes L, Casasnovas JM. 2012. Structural Bases of Coronavirus Attachment to Host Aminopeptidase $\mathrm{N}$ and Its Inhibition by Neutralizing Antibodies. Plos Pathogens, 8: e1002859.

Schwegmann-Wessels C, Glende J, Ren XF, Qu XX, Deng HK, Enjuanes L, Herrler G. 2009. Comparison of vesicular stomatitis virus pseudotyped with the $\mathrm{S}$ proteins from a porcine and a human coronavirus. J Gen Virol, 90: 1724-1729.

Shahwan K, Hesse M, Mork AK, Herrler G, Winter C. 2013. Sialic Acid Binding Properties of Soluble Coronavirus Spike (S1) Proteins: Differences between Infectious Bronchitis Virus and Transmissible Gastroenteritis Virus. Viruses-Basel, 5: 19241933.

Tang DJ, Lam YM, Siu YL, Lam CH, Chu SL, Peiris JSM, Buchy P, Nal B, Bruzzone R. 2012. A Single Residue Substitution in the Receptor-Binding Domain of H5N1 Hemagglutinin Is Critical for Packaging into Pseudotyped Lentiviral Particles. Plos One, 7: e43596.

Tusell SM, Schittone SA, Holmes KV. 2007. Mutational analysis of aminopeptidase $\mathrm{N}$, a receptor for several group 1 coronaviruses, identifies key determinants of viral host range. J Virol, 81: 1261-1273.

Wu KL, Chen L, Peng GQ, Zhou WB, Pennell CA, Mansky LM, Geraghty RJ, Li F. 2011. A virus-binding hot spot on human angiotensin-converting enzyme 2 is critical for binding of two different coronaviruses. J Virol, 85: 5331-5337. 